\title{
Self-recovery, Fatigue and Anti-fatigue of Supramolecular Elastomers
}

\author{
A.D. Drozdov ${ }^{1 *}$ and J. deClaville Christiansen ${ }^{1}$
}

1) Aalborg University, Aalborg, Denmark
${ }^{*}$ E-mail aleksey@m-tech.aau.dk

Supramolecular elastomers (SMEs) are three-dimensional networks of polymer chains bridged by covalent cross-links and non-covalent bonds with finite lifetimes. The reversible noncovalent bonding is based on (i) metal-ligand coordination, (ii) electrostatic interaction, (iii) hydrophobic association, (iv) hydrogen bonding, and (v) host-guest recognition. An advantage of these materials compared with conventional vulcanized rubbers and thermoplastic elastomers is their ability of rapid self-healing after damage at ambient temperature. The mechanical response of SMEs is characterized by (i) rapid relaxation of stresses (with the characteristic time of a few minutes), (ii) self-recovery (a monotonic reduction in plastic strain with time after cyclic loading), and (iii) anti-fatigue (an increase in maximum stress with number of cycles under deformation programs with intervals of recovery between cycles).

A constitutive model is developed for the viscoelastic and viscoplastic behavior of supramolecular elastomers. Stress-strain relations are derived from the free energy imbalance inequality for an arbitrary three-dimensional deformation with finite strains. The viscoelastic response reflects breakage and reformation of temporary bonds in a transient polymer network (transition of chains from their active to dangling state and vice versa). The viscoplastic response reflects slippage of permanent junctions with respect to their reference positions. The model is applied to fit experimental data in tensile loading-unloading tests, relaxation tests, and multi-cycle tests on several SMEs. Numerical simulation shows that the governing equations describe adequately the experimental stress-strain diagrams, the material parameters evolve consistently with chemical composition of SMEs and experimental conditions, and predictions of the model are in agreement with observations.

Financial support by the Danish Innovation Fund (project 5152-00002B) is gratefully acknowledged.
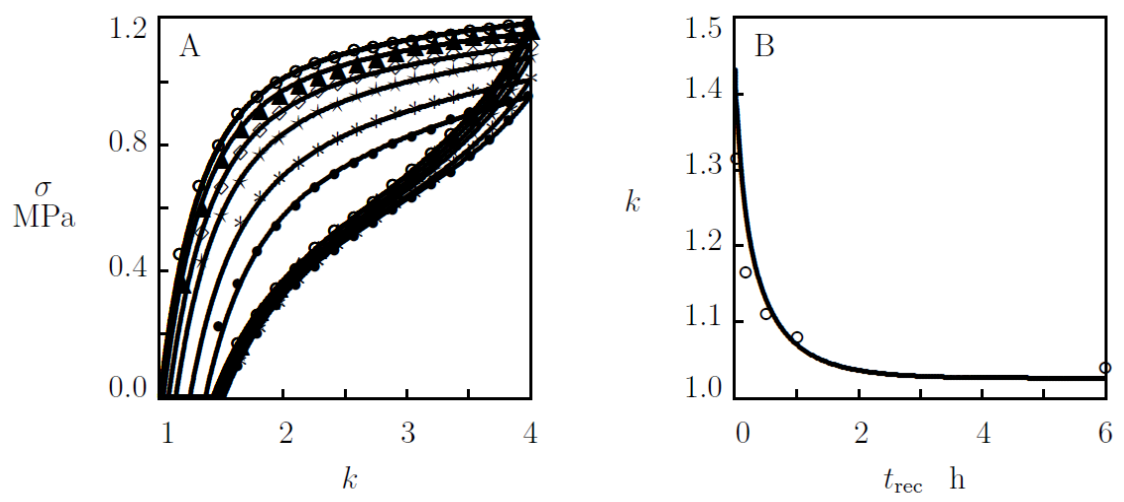

Figure 1. Self-recovery of PB-TAA elastomer. Symbols: experimental data. Solid lines: results of simulation. 